\title{
License Status
}

National Cancer Institute

\section{Source}

National Cancer Institute. License Status. NCI Thesaurus. Code C118405.

The standing or condition of the license of an individual or business. 